\title{
Exciting High Frequency Oscillations in a Coaxial Transmission Line with a Magnetized Ferrite
}

\author{
J.-W. Ahn ${ }^{1 *}$, S. Y. Karelin' ${ }^{2}$ H.-O. Kwon ${ }^{1}$, I. I. Magda ${ }^{2}$, and V. G. Sinitsin ${ }^{2}$ \\ ${ }^{1}$ Hanwha Corp., Gumi, Kyungbuk 39370, Korea \\ ${ }^{2}$ National Science Center 'Kharkov Institute of Physics and Technology', Kharkov 61108, Ukraine
}

(Received 22 October 2015, Received in final form 24 November 2015, Accepted 24 November 2015)

\begin{abstract}
The paper presents methodologies and results concerning one- and two-dimensional numerical modeling of radio frequency oscillations in a coaxial transmission line fed with a short pulse of electric current. The line is partially filled with a ferrite material, magnetized longitudinally close to saturation. The 2D model has permitted analyzing, for the first time in the art, the spatial structure and dynamics of the wave field within the radially non-uniform cross-section planes of the non-linear and dispersive guiding structure. This opens ways for optimizing size parameters of the line and the extent to which it is filled with the ferromagnetic material, thus increasing the line's electric strength and intensity of the r.f. oscillations.
\end{abstract}

Keywords : pulsed electric current, saturated ferrite, quasi-monochromatic oscillations

\section{Introduction}

The non-linear effects accompanying propagation of pulsed electric currents through a transmission line partially filled with a ferro- or ferrimagnetic material have been studied since early 1960s [1-8], first in connection with pulse sharpening and formation of electromagnetic shocks $[1,2]$. Today, the attention of researchers has shifted toward direct conversion of the short video pulse into radio frequency (rf) electromagnetic oscillations which can be extracted from the generating structure in the form of high power pulsed radiation [3-8]. High power electromagnetic pulses of nanosecond duration prove useful in a variety of applications, such as digital data transmission, energy transfer, radar or medical/ biological research [9]. The emerging techniques of dc-torf conversion represent a promising alternative to the microwave generators based on intense electron beams. In this paper the formation of quasi-monochromatic oscillations in a ferrite-filled nonlinear transmission line (NLTL) is analyzed by means of numerical modeling based on 1D and 2D theoretical models. The results are compared with experiment.

CThe Korean Magnetics Society. All rights reserved.

*Corresponding author: Tel: $+82-54-467-8808$

Fax: +82-54-467-8780, e-mail: jwahn@hanwha.com

\section{Experiment}

The structure that has been tested experimentally and simulated numerically (Fig. 1) involved a linear coaxial transmission line, TL1, which the initial video pulse is fed through; a ferrite-filled line, NLTL, exhibiting nonlinear and dispersive properties, and another linear line, TL2 for signal extraction. The inner and the outer conductors (diameters $D_{1}$ and $D_{3}$, respectively) are the same for all the three lines. The ferrite cylinder (set of rings of diameter $D_{2}$ ) is separated from the outer metal conductor by an insulating layer (indicated as Dielectric).

The oscillations are expected to appear in the NLTL when the ferrite reaches a magnetic state close to saturation. The material selected for our preliminary experiment was the high-frequency NiZn ferrite of the 'soft' grade 200ВНП, with which previous writers [5-7] reportedly had obtained the most satisfactory results. The parameters

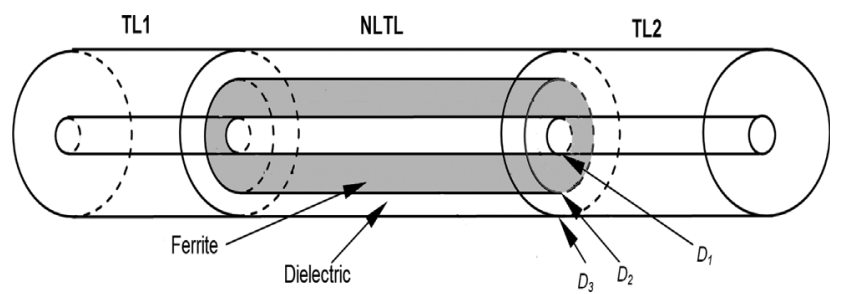

Fig. 1. Schematic of the coaxial structure with a nonlinear, dispersive transmission line NLTL. 
of the structure were as follows,

- electrode diameters of the coaxial transmission lines TL1, NLTL and TL2, $D_{1}=12 \mathrm{~mm}$ (inner) and $D_{3}=26$ $\mathrm{mm}$ (outer);

- full length of the set of transmission lines, $\Lambda=2550$ $\mathrm{mm}$;

- sizes of the ferrite insert, respectively the inner and the outer diameter, $D_{1}=12 \mathrm{~mm}$ and $D_{2}=20 \mathrm{~mm}$, and length $l=800 \mathrm{~mm}$;

- relative dielectric constants of the ferrite and the liquid dielectric (transformer oil), $\varepsilon_{f}=10$ and $\varepsilon_{d}=2.25$, respectively.

An external 'bias' magnetic field $\boldsymbol{H}_{\mathbf{0}}$ was applied along the axis of the transmission line to bring the ferrite in the NLTL closer to saturation. The pulsed current contributed to transverse components of the magnetic field and was involved in non-linear interactions with the anisotropically magnetized ferrite. The results could be sharpening of the front edge and appearance of radio frequency oscillations. Shown in Fig. 2 are pulsed waveforms as measured with voltage sensors at the input and output of the above described NLTL. Upon interaction with the magnetized ferrite the pulsed signal can be seen to have acquired a much shorter front edge (about $0.2 \mathrm{~ns}$ in width against the initial 3.5 to $4 \mathrm{~ns}$ ) and an oscillatory component.

Quasi-monochromatic oscillations of relative amplitude $\Delta U / U_{0}<27 \%$ and frequency $1.58 \mathrm{GHz}$ (quasi-period $0.63 \mathrm{~ns}$ ) were obtained for $U_{0}=100 \mathrm{kV}$ and $H_{0}=96 \mathrm{kA} /$ $\mathrm{m}$, where $U_{0}$ is the initial peak magnitude of the applied voltage pulse.
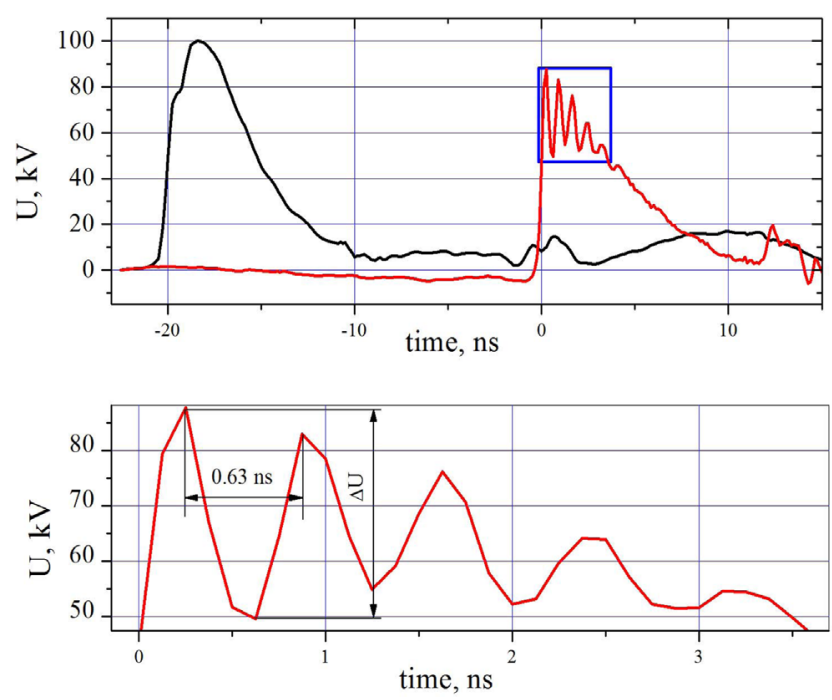

Fig. 2. (Color online) Voltage waveforms in the NLTL with $H_{0}=96 \mathrm{kA} / \mathrm{m}$ : sensors at the line input (black curve) and at the output (red-colored curve). The lower panel shows details of the inset (the oscillating component of full span $\Delta U$ ).

\section{Theory and Models}

The propagation of electromagnetic waves in the coaxial structure of Fig. 1 can be described within several theoretical models whose reality and complexity are determined by the structural features and physical effects taken into account. All of the models proceed from the set of Maxwell equations written for the cylindrical frame of reference (coordinates $\rho, \varphi, z$ for the radial, azimuthal and axial directions, respectively; SI units adopted),

$$
\begin{aligned}
& \varepsilon_{0} \frac{\partial\left(\varepsilon_{r} E_{\rho}\right)}{\partial t}=\frac{1}{\rho} \frac{\partial H_{z}}{\partial \varphi}-\frac{\partial H_{\phi}}{\partial z} \\
& \varepsilon_{0} \frac{\partial\left(\varepsilon_{\mathrm{r}} E_{z}\right)}{\partial t}=-\frac{1}{\rho} \frac{\partial H_{\rho}}{\partial \varphi}+\frac{1}{\rho} \frac{\partial\left(\rho H_{\phi}\right)}{\partial \rho} ; \\
& \varepsilon_{0} \frac{\partial\left(\varepsilon_{\mathrm{r}} E_{\phi}\right)}{\partial t}=\frac{\partial H_{\rho}}{\partial z}-\frac{\partial H_{z}}{\partial \rho} \\
& \mu_{0} \frac{\partial\left(H_{\rho}+M_{\rho}\right)}{\partial t}=\frac{\partial E_{\phi}}{\partial z}-\frac{1}{\rho} \frac{\partial E_{z}}{\partial \varphi} \\
& \mu_{0} \frac{\partial\left(H_{\phi}+M_{\phi}\right)}{\partial t}=\frac{\partial E_{z}}{\partial \rho}-\frac{\partial E_{\rho}}{\partial z}
\end{aligned}
$$

and

$$
\mu_{0} \frac{\partial\left(H_{z}+M_{z}\right)}{\partial t}=\frac{1}{\rho} \frac{\partial E_{\rho}}{\partial \varphi}-\frac{1}{\rho} \frac{\partial\left(\rho E_{\phi}\right)}{\partial \rho},
$$

with incorporated constituent relations $\boldsymbol{D}=\varepsilon_{0} \varepsilon_{\mathrm{r}} \boldsymbol{E}$ and $\boldsymbol{B}=$ $\mu_{0}(\boldsymbol{H}+\boldsymbol{M})$. Here $\boldsymbol{D}, \boldsymbol{E}, \boldsymbol{H}$ and $\boldsymbol{B}$ are field vectors, and $\boldsymbol{M}$ the magnetization vector, or per unit volume magnetic moment in the magnetic material; $\varepsilon_{0}$ and $\mu_{0}$ are the permittivity and permeability of free space, and $\varepsilon_{r}$ the relative permittivity of an electrically isotropic medium. In the lines TL 1 and TL 2 containing no ferrite, $\boldsymbol{M}=0$. The dispersive, anisotropic and nonlinear properties of the ferrite material are taken into account phenomenologically through the equation of motion for $\boldsymbol{M}$ (Landau-Lifshitz equation) [10],

$$
\frac{d \boldsymbol{M}}{d t}=\gamma \mu_{0}[\boldsymbol{M} \times \boldsymbol{H}]-\alpha \frac{\gamma \mu_{0}}{M_{s}}[\boldsymbol{M} \times[\boldsymbol{M} \times \boldsymbol{H}]],
$$

where $\gamma$ stands for electron's gyromagnetic ratio; $\boldsymbol{H}$ is the effective 'internal' magnetic field in the ferrite, and $\alpha$ denotes a certain relaxation coefficient. It is assumed that the ferrite has been brought close to a saturated state, $|\boldsymbol{M}|=M_{s}$, by application of a sufficiently high de bias field $\boldsymbol{H}_{0}=\mathbf{e}_{\mathbf{z}} H_{0}$, such that $\boldsymbol{H}=\boldsymbol{H}_{0}+\boldsymbol{h}$ and $\boldsymbol{M}=\boldsymbol{e}_{z} M_{s}+\boldsymbol{m}$ in all the equations.

\subsection{D model}

The great majority of previous papers on the subject 
(e.g., $[3-5,8])$ made use of the simple model where the electromagnetic pulse in the ferrite retained the initial modal structure of a TEM wave, as supported by the 'empty' TL1 line. Equations (1) and (5), with $\partial / \partial \varphi=0$ and $\partial / \partial \rho=0$, and involving the only non-zero field components $E_{\rho}, E_{\varphi}$ and $H_{z}$, then could be recast to familiar telegraph equations that govern the voltage and current in the coaxial line conductors, and the magnetic flux through the cross-section plane [2-4]. These, in turn, could be brought to a second-order differential equation for the current $I$ through the line (alternatively, voltage), viz.

$$
\frac{\partial^{2} I}{\partial z^{2}}=L C \frac{\partial^{2}\left(I+K \cdot m_{\varphi}\right)}{\partial t^{2}}+\frac{1}{C} \frac{\partial C}{\partial z} \frac{\partial I}{\partial z}-R C \frac{\partial I}{\partial t},
$$

where $C$ and $L$ are, respectively, the per unit length capacitance and inductance of the 'empty' line without a ferromagnetic filling; $R$ the per unit length ohmic resistance; $m_{\varphi}$ the azimuthal component of ferrite magnetization which is to be found from the Landau-Lifshitz equation (7) as a function of $\boldsymbol{h}$, and $K$ a numerical coefficient to couple the magnetic flux due to the current pulse to that from the bias field. Obviously enough, this approach does not allow analyzing variations of the electromagnetic field along the coordinates transverse to the propagation direction, $z$, similarly as it ignores polarization of the field, while making account of the ferrite's non-linear response. Dolan's paper [3] was among the best physically grounded $1 \mathrm{D}$ analyses of pulse propagation through the NLTL, yet its account of the effective field in the ferrite (in terms of classic demagnetizing factors) seemed to be fully empirical rather than self-consistent (and, apparently, remained within a linear approximation). The corresponding estimates for $h_{\rho}, h_{\varphi}$ and $h_{z}$ were

$$
h_{\rho}=-m_{\rho} ; h_{\varphi}=q I \text {, and } h_{\mathrm{z}}=-k\left(m_{\mathrm{z}}-M_{\mathrm{s}}\right), 0<k<1 \text {, }
$$

with $q=1 / \pi d_{e f f}, d_{e f f}=\left(D_{2}-D_{1}\right) / \log \frac{D_{2}}{D_{1}}$, and

$K=\mu_{0} \frac{D_{2}-D_{1}}{2 l}$.

In this work we have also appealed, at an early stage, to the 1D model and used field estimates similar to Eq. (9) but, in contrast to [2-4] and other papers, obtained these through a joint numerical solution of the Landau-Lifshitz (L-L) equation (7) and the telegraph equation (8). Besides, our computations covered the entire coaxial structure rather than the NLTL alone, which enabled us to consider the questions of pulse reflection and line matching at the TL1-NLTL-TL2 transitions.

To jointly solve Equations (7) and (8), an $n \times N$ mesh was formed with $n=\Lambda / \Delta z$ and $N=T / \Delta t$, where $\Lambda$ is the full length of the coaxial structure; $T$ the computation time, and $\Delta z$ and $\Delta t$ are discretization steps in length and time, respectively. Each cell of the mesh is associated with a current of magnitude $I_{\mathrm{i}, j}$ and magnetic moment vector $\boldsymbol{m}_{\mathrm{i}, j}$ with components $\left(m_{\rho}\right)_{i, j},\left(m_{\varphi}\right)_{i, j}$ and $\left(m_{z}\right)_{i, j}$, where $i=1,2, \ldots n$, and $j=1,2 \ldots N$.

The computations at each time step were done in the format of three sub-problems,

i) prediction of the net magnitude of $I_{\mathrm{S}}=I_{i+1, j}+\left(m_{\varphi}\right)_{i+1, j}$ for time moment $i+1$ (next after moment $i$ ) through numerical solution of the telegraph equation (8) in the finite-difference technique. The individual components of $I_{\mathrm{S}}$ for moment $i+1$ will not be known yet;

ii) evaluation of the magnetic moment $m_{\varphi}$ for time moment $i+1$ through solution of the L-L equation (7) by the Runge-Kutta method;

iii) evaluation of the current magnitude proper for time moment $i+1, I_{i+1, j}=I_{\mathrm{S}}-K \times\left(m_{\varphi}\right)_{i+1, j}$.

The computations have been done for a few configurations of the coaxial structure and a variety of ferrite

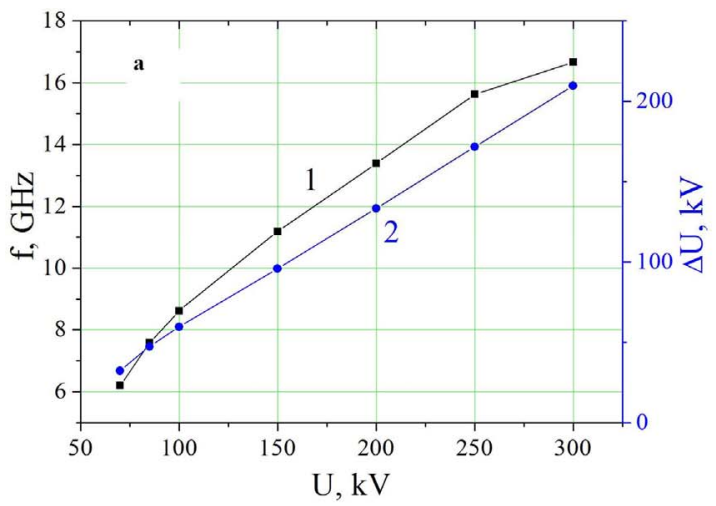

Fig. 3. (Color online) 1D model: The oscillation frequency, $\mathrm{f}$ (black curves 1 in panels a, b), and amplitude, $\Delta U$ (blue curves 2 in $\mathrm{a}, \mathrm{b}$ ) as functions of the input pulsed voltage, $U$, with $H_{0}=100 \mathrm{kA} / \mathrm{m}$ (panel a) and of the longitudinal magnetizing field, $H_{0}$, with a current-induced azimuthal field $H_{\varphi}=110 \mathrm{kA} / \mathrm{m}$ (panel b). 

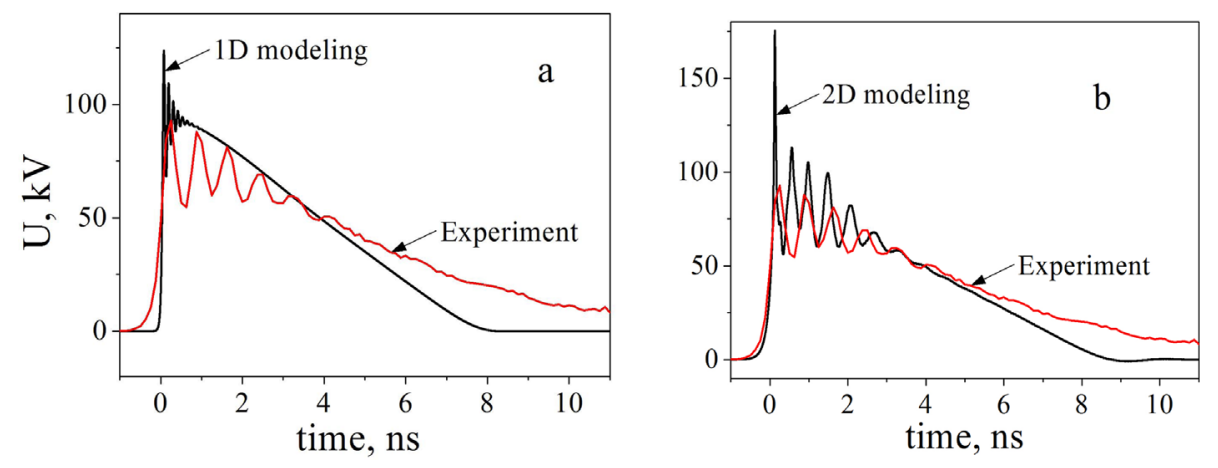

Fig. 4. (Color online) Comparison of the output signals obtained in the experiment, and in 1D (a) and 2D (b) simulations.

parameters, as well as waveforms of the injected current pulses. The results relative the line configuration and pulse parameters described in Section1 are presented in Figs. 3 and 4.

As can be seen from Fig. 3, the oscillation frequency increases with the input voltage and decreases with the longitudinal magnetizing field. The tendencies are in agreement with the experimental data of, for example, paper [3], suggesting a qualitative correctness of the 1D model. Meanwhile, the frequency obtained in the 1D numerical experiment happens to be much higher than either was reported in [3] or observed in our experiment (see Fig. 4, a). This means that the 1D model is not, in fact, a fully adequate representation of the real coaxial structure where the filling material is an anisotropic medium, and the electromagnetic field components (in a greater number than three) are not uniformly distributed along the radial coordinate. The problem requires a treatment in a higher dimension.

\subsection{D model}

A two-dimensional model is obtained from the full set of Maxwell equations (1-6) by setting $\partial / \partial \varphi=0$, while $\partial / \partial \rho \neq 0, \partial / \partial z \neq 0$, in view of the initial azimuthal symmetry of the problem. The solutions are identified as TE and TM modes, where the TE wave is in a sense close to the TEM mode that existed in the NLTL in the 1D case and now remains excited in the empty TL1 line. The TM wave appears as a result of mode coupling owing to the magnetic anisotropy (i.e., the magnetic moment's equation of motion (7)). The Maxwell equations with $\partial / \partial \varphi=0$, plus the L-L equation (7) have been solved jointly within a finite-element time domain technique [11], following the same logic as in the 1D case to account for the interaction between the electromagnetic fields and the magnetic subsystem. (Once again, the development of every field vector with time in each spatial cell of the computational domain was followed in terms of the three differential-difference sub-problems).

\section{Results and Discussion}

The results of numerical simulation are shown in Fig. 4-Fig. 8. As can be seen from Figs. 4 and 5, the 2D modeling yields a pulsed waveform at the output from the NLTL that corresponds much better to the experimental result than the 1D model. In particular, the estimate for the oscillation frequency $(2.05 \mathrm{GHz})$ is really closer to the
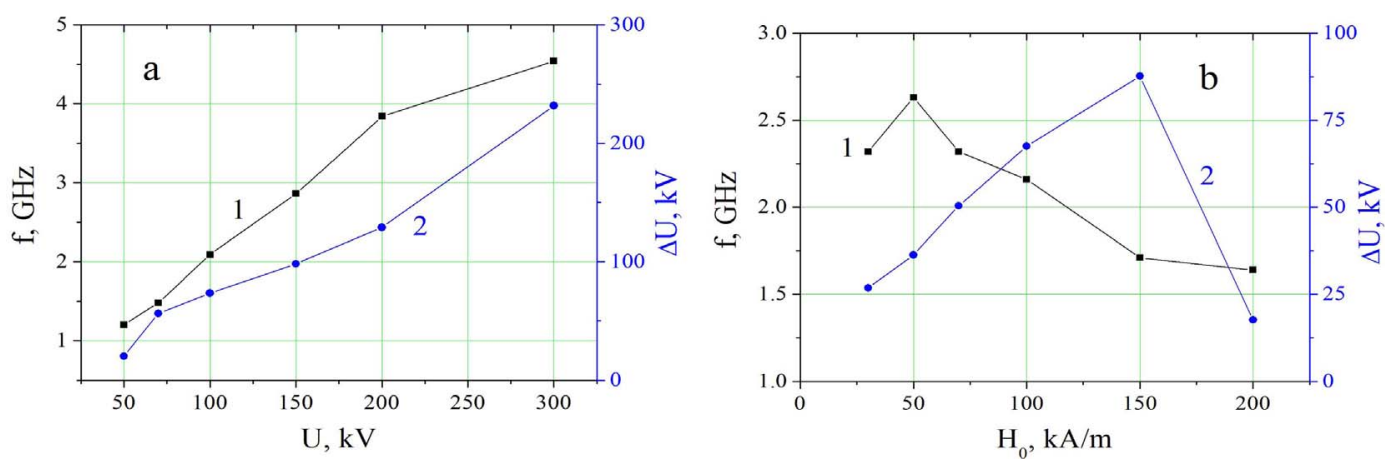

Fig. 5. (Color online) 2D model: The oscillation frequency, $f$ (black curves 1 in a, b), and amplitude, $\Delta U$, (blue curves 2 in a, b) as functions of the input voltage, $U$, with $H_{0}=100 \mathrm{kA} / \mathrm{m}$ (panel a) and of the longitudinal magnetizing field, $H_{0}$, with a currentinduced azimuthal field $H_{\varphi}=110 \mathrm{kA} / \mathrm{m}$ (panel b). 

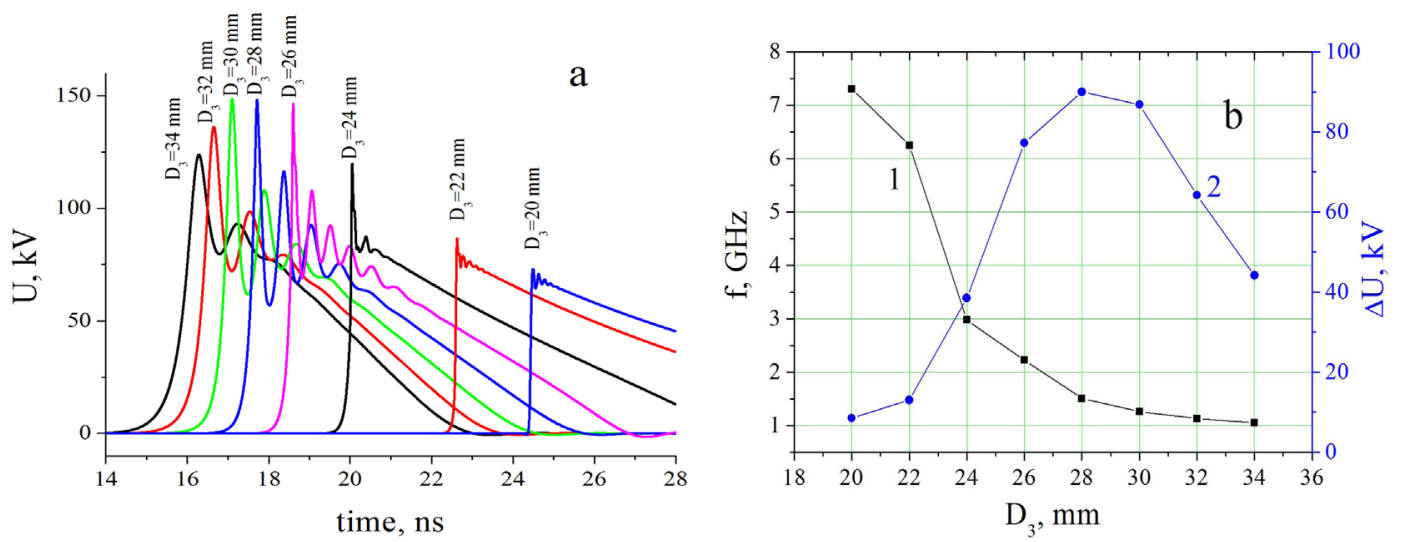

Fig. 6. (Color online) The output pulse waveform (panel a) oscillation frequency (panel b, black curve 1) and oscillation amplitude (panel b, blue curve 2) versus the NLTL outer diameter, $D_{3}$.

value measured in the experiment $(1.6 \mathrm{GHz})$ than the $1 \mathrm{D}$ result $(8.5 \mathrm{GHz})$.

Proceeding from the 2D numerical study described, we are in a position to consider a few issues of practical interest, namely

(i) Optimized value for the filling factor of the ferritecontaining NLTL. To study this, a series of numerical experiments have been conducted with various outer diameters $D_{3}$ of the structure (Fig. 1), seeking optimization in terms of a maximum amplitude for the rf oscillations. A maximum of the kind can be established in Fig. 6 near $D_{3}=28 \mathrm{~mm}$, which diameter corresponds to a filling factor about 0.4 across the waveguide's cross-section. If we varied the filling factor independently, then upon approaching unity it would raise the oscillation frequency to as high magnitudes as 5 to $6 \mathrm{GHz}$, i.e. roughly the results of the 1D model. However with a decreasing filling factor the oscillation frequency also decreases rapidly.

This means that the frequency of rf oscillations in the radially layered structure of the NLTL depends on the transverse distribution of the electromagnetic field (that could not be accounted for within the 1D model).

(ii) Transverse dimensions of the NLTL. To increase the power of the rf oscillations one needs increasing the input pulsed voltage $U_{0}$. Then, to ensure a proper electrical strength it is necessary to increase the cross-sectional size of the coaxial structure. However, increasing the transverse dimensions necessarily leads to appearance of the effects peculiar to layered waveguides, specifically conversion of the TEM wave into TE and TM modes, and, as a result, distortion of the output pulse. To explore the issue, a few numerical experiments were done where the input voltage $U$ and the coaxial guide's diameter $D$ were varied proportionally, i.e. $U=s U^{0}$ and $D_{i}=s D_{i}^{0}$, with $s$ being a variable scaling factor, The super-script ${ }^{0}$ relates to some set initial values of both $U$ and $D$. Fig. 7, a presents a series of the output waveforms, obtained for the driving signal of amplitude $U^{0}=100 \mathrm{kV}$ and half-maximum pulse width $t_{p 0.5}=6 \mathrm{~ns}$. The reduced span $\Delta U / \mathrm{s}$ of the oscillation amplitude is seen to increase at larger values of $s, e$.g. up
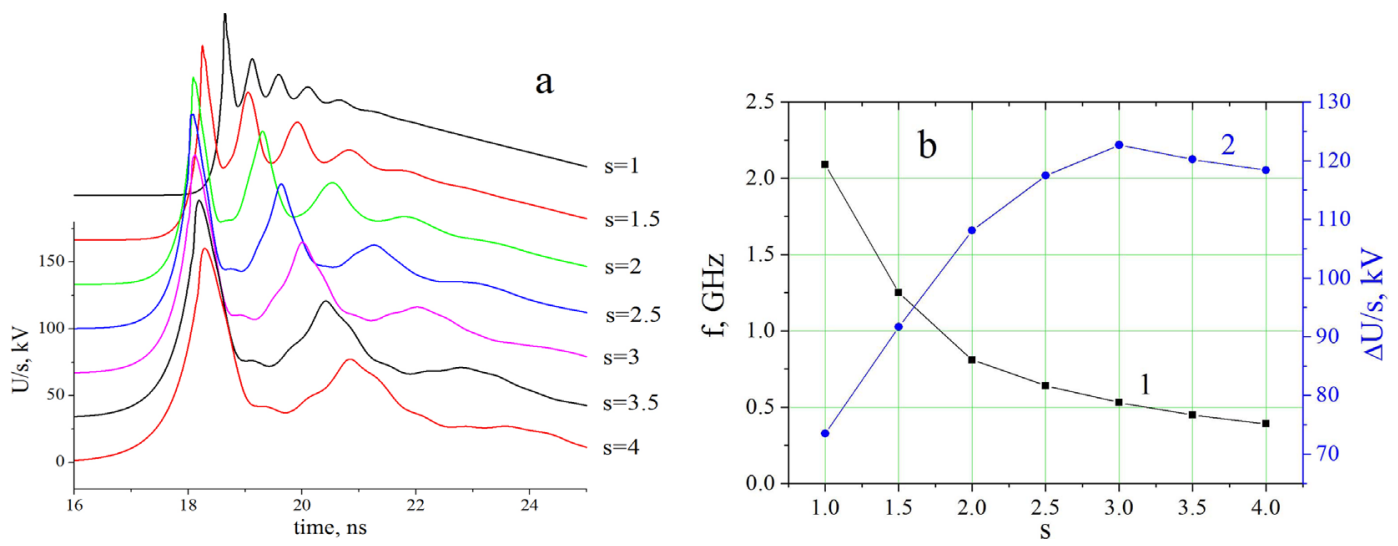

Fig. 7. (Color online) Voltage waveforms at the output from the NLTL as functions of the scaling parameter, $s$ (panel a), and the dependences upon $s$ (panel b) shown by the oscillation frequency (curve 1, black) and reduced amplitude span (curve 2). 

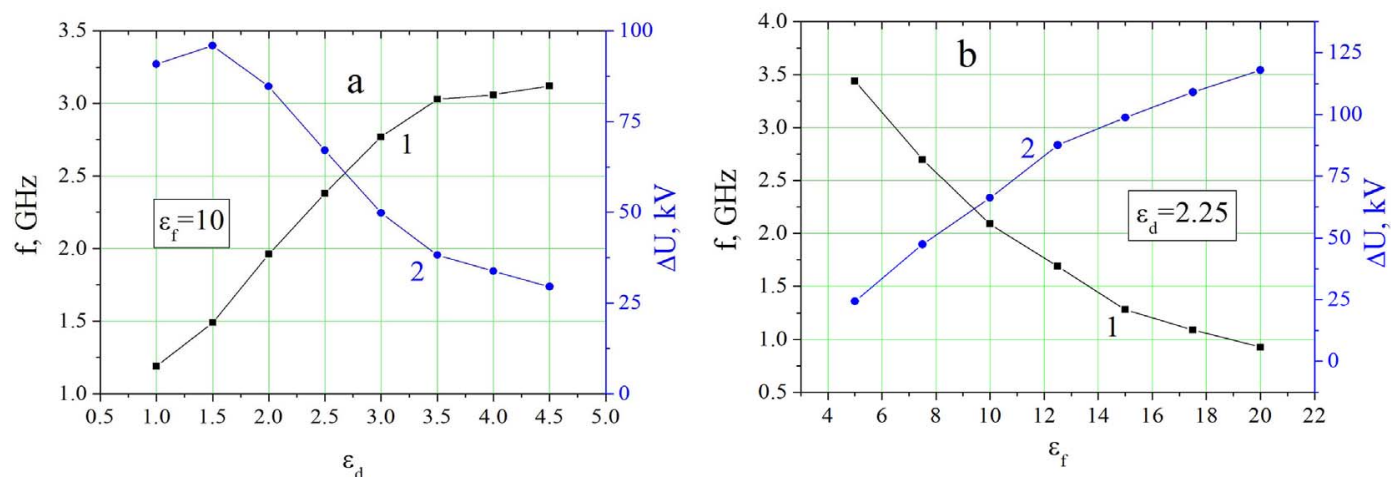

Fig. 8. (Color online) Dependences of the oscillation frequency (black curves 1 in a and b), and amplitude span (blue curves 2) on relative electric permittivities of the liquid dielectric (panel a) and ferrite material (panel b).

to $s=3$.

It should be noted, however, that the oscillation frequency drops down with an increase of $s$ (an effect that was absent in the 1D model), similar as the number of the oscillation periods observed, because the width of the current pulse is limited. In fact, at greater values than $s=3$ the oscillations practically disappear (at least with $t_{p 0.5}=6 \mathrm{~ns}$ ), such that no more than 1 or 2 periods can be observed, $c f$. Fig. 7. With greater pulse widths of the driving current the oscillations are observable at lower frequencies.

iii) The effect of dielectric characteristics of the NLTL media on the excitation efficiency of oscillations. A series of numerical experiments with variations in the permittivity of the liquid dielectric, $\varepsilon_{d}$, and of the ferrite material, $\varepsilon_{f}$, were performed. These results are shown in Fig. 8. As can be seen, when $\varepsilon_{f}$ decreases and $\varepsilon_{d}$ increases, the oscillation frequency grows up, while the oscillation amplitude goes down. Thus, to ensure an efficient formation of HF oscillations the permittivity of the ferrite should be significantly higher than such of the insulating dielectric. By way of example, for a coaxial NLTL with dimensions $D_{3} / D_{1}=2.2$, excited by an input pulse of amplitude 100 $\mathrm{kV}$ and a 6 ns pulse width the conditions for effective excitation of oscillations can be formulated as follows,

- the factor of filling the line's cross-section with the ferrite material should be about 0.4 ;

- the permittivity of an insulating dielectric should be at least three times lower than the permittivity of the ferrite, $\varepsilon_{f}>3 \varepsilon_{d}$.

\section{Conclusions}

A 2D simulation model has been used for the first time in the art to describe the dynamics of the shock wave and the HF oscillations excited by a current impulse traveling along a nonlinear coaxial line partially filled with a longitudinally magnetized ferrite. The 2D model demonstrates major advantages over the 1D model and agrees much better with experimental results. The computations based on the 2D model allow formulating practical conditions for an effective formation of HF oscillations in the NLTL.

\section{References}

[1] A. M. Belyantsev, Y. K. Bogatyryov, and L. I. Solovyova, Izv. VUZ-Radiofizika, 6, 551 (1963).

[2] I. G. Katayev, Electromagnetic shock waves, Illife Books, London (1966), pp. 1-50.

[3] J. E. Dolan, J. Phys. D: Appl. Phys. 32, 1826 (1999).

[4] A. M. Belyantsev, A. I. Dubnev, I. Klimin, Y. A. Kobelev, and L. A. Ostrovsky, Techn. Phys. 40, 820 (1995).

[5] V. P. Gubanov, A. V. Gunin, O. B. Kovalchuk, V. O. Kutenkov, I. V. Romanchenko, and V. V. Rostov, Techn Phys Letters 35, 42 (2009).

[6] I. V. Romanchenko, V. O. Kutenkov, and V. V. Rostov, IEEE 36th International Conference on Plasma Science, San-Diego, CA, 1 (2009).

[7] V. V. Rostov, N. M. Bykov, D. N. Bykov, A. I. Klimov, O. B. Kovalchuk, and I. V. Romanchenko, IEEE Trans. Plasma Sci. 38, 2681 (2010).

[8] D. V. Reale, PhD dissertation, Texas Tech University, TX, USA (2013).

[9] A. V. Gaponov-Grekhov and V. L Granatstein, Applications of high-power microwaves, Artech House, Boston (1994).

[10] L. Landau and E. Lifshitz, Physik. Z. Sowietunion 8, 153 (1935).

[11] A. Taflove and S. C. Hagness, Computational Electrodynamics: The Finite-Difference Time-Domain Method, Artech House, Boston-London (2000). 